\title{
Medidas de Desempeño Logístico: Una Revisión
}

\author{
Logistics Perfomance Tracking: a Review
}

Orlando Zapateiro-Altamiranda

Escuela Naval de Cadetes "Almirante Padilla" -

Colombia

ORCID iD: https://orcid.org/0000-0003-4567-9814

ozapateiro@hotmail.com
Fecha de recepción: 16/03/2020

Fecha de evaluación: 09/05/2020

Fecha de aceptación: 10/06/2020

Cómo citar: Zapateiro-Altamiranda, O. (2020). Medidas de Desempeño Logístico: Una Revisión. Revista Científica Anfibios, 3(2), 76-82. https://doi.org/10.37979/afb.2020v3n2.75

\section{Resumen}

Con los retos organizacionales que han propuesto fenómenos como la globalización, clientes cada vez más exigentes y la digitalización de productos y servicios, es necesario poner a disposición de estas organizaciones modelos de gestión logística que puedan responder ante tales desafíos. En ese orden de ideas y por medio de una revisión de la literatura analítica, se exponen las siguientes medidas de desempeño logístico; la Medición de indicadores de desempeño de niveles estratégico, táctico y operativo de Gunasekaran; la medición de indicadores de desempeño en los procesos de la cadena logística; el modelo de SCOR (Supply Chain Operations Reference); y el modelo de referencia de logístico (LMR) de Donald Bowersox, comentando sus principales características de tal manera que tanto académicos como quienes toman las decisiones en el área logística al interior de las organizaciones tengan una herramienta cognoscitiva que les permita elegir el modelo que se adapte a sus necesidades. Los hallazgos principales se vinculan con una descripción sucinta de los modelos logísticos, haciendo aportes en lo académico y en lo práctico, exhortando a futuras investigaciones para que profundicen en mayor medida estas medidas del ámbito logístico organizacional.

\section{Palabras clave:}

Medidas de desempeño logístico; gestión de cadena de suministro; indicadores logísticos; modelos logísticos; organizacional.

\begin{abstract}
With the organizational challenges posed by phenomena such as globalization, increasingly demanding clients and the digitalization of products and services, it is necessary to make available to these organizations logistics management models that can respond to such challenges. In that order of ideas and through a review of the analytical literature, the following logistics performance measures are presented: Gunasekaran's Measurement of Performance Indicators of Strategic, Tactical and Operational Levels; Measurement of Performance Indicators in the processes of the logistics chain; SCOR (Supply Chain Operations Reference) model; and Donald Bowersox's Logistics Reference Model (LMR), commenting on its main
\end{abstract}


characteristics so that both academics and decision makers in the logistics area within organisations have a cognitive tool that allows them to choose the model that suits their needs. The main findings are linked to a succinct description of the logistics models, making contributions in the academic and practical areas, encouraging future research to go deeper into these measures of the organizational logistics field.

\section{Keywords:}

Logistics performance measures; supply chain management; logistics indicators; logistics models; organizational

\section{Introducción.}

El fenómeno de la globalización ha llevado a las organizaciones a un estado de búsqueda constante de competitividad, pasando de ventajas comparativas a ventajas competitivas (Pinheiro de Lima et al., 2017), debido a esto, las variables externas, necesidades y requerimientos cada vez más exigente por parte de los mercados, hacen necesario que se cuantifique el impacto y la evolución de los factores que involucran las actividades importantes de las organizaciones (Arango Serna et al., 2017) ello se logra mediante el manejo logístico de la información, ya que mediante diversas herramientas se obtienen datos estratégicos (Fram \& Licona, 2016). En ese marco, la cadena logística ha cobrado gran importancia desde la década de 1980's debido a que se ha consolidado como una fuente importante de ventajas competitivas (Orjuela Castro et al., 2016) en la que se involucran características como; eficacia; eficiencia; agilidad; dinamismo; creatividad; flexibilidad; logrando una visión del panorama general del negocio, aumenten la competitividad y hayan desarrollen estrategias que posibiliten la sostenibilidad empresarial (Pinheiro de Lima et al., 2017).

Bajo este contexto, la literatura vinculada con la cadena de abastecimiento ha logrado proponer diversos modelos de desempeño logístico a lo largo de su tiempo de estudio, proporcionando las herramientas para que las organizaciones en la actualidad puedan aumentar su desempeño generando ventajas competitivas desde sus procesos logísticos (Del Río et al., 2009). Es por esto que el objetivo del presente artículo es exponer algunas medidas de desempeño logístico con el objetivo de acercar estos modelos tanto a la academia como a los tomadores de decisiones al interior de las or- ganizaciones y de esa manera promover la investigación en este campo y a al mismo tiempo la generación de las ventajas competitivas al interior de las organizaciones como principales aportes, además de plantear algunas discusiones respecto a otros ejes temáticos en donde se pueden utilizar las herramientas propuestas.

Es de esta manera que se proponen tres secciones para abordar los planteamientos acerca de las medidas de desempeño logístico. Inicialmente se plantea un apartado teórico que permite conectar las necesidades que tienen las organizaciones, tomando en cuenta los retos a los que se enfrenta en la actualidad, con los modelos logísticos de tal manera que estos fungen como una alternativa que permite a las organizaciones responder ante estos desafíos y de esa manera lograr competitividad. Posteriormente se presenta el diseño metodológico para presentar como se llegó a cabo la revisión y los criterios de inclusión y exclusión utilizados. Seguidamente se presentan formalmente los cuatro modelos de gestión logística al interior de las organizaciones; la Medición de indicadores de desempeño de niveles estratégico, táctico y operativo de Gunasekaran; la medición de indicadores de desempeño en los procesos de la cadena logística; el modelo de SCOR (Supply Chain Operations Reference); y el modelo de referencia de logístico (LMR) de Donald Bowersox.

Finalmente se plantean conclusiones, entre las que resalta el aporte a las dinámicas del desempeño logístico otorgando una visión holística del panorama de medidas de desempeño en esas áreas, con la finalidad de ofrecer alternativas para que las organizaciones tomen decisiones respecto a cuál modelo implementar. Adicionalmente, se proponen discusiones entre las que se sobresalen la utilidad de las 
medidas de desempeño logístico revisadas en otros ámbitos además del organizacional, es decir, evaluando el desempeño logístico en entes territoriales, promoviendo así su desarrollo económico, o la generación de valor en economías emergentes.

\section{Abordaje Teórico}

Para Pinheiro de Lima et al., (2017) la logística, o también llamada "Administración de la logística" se encuentra asociada con características de las organizaciones como; el tráfico y medios de transporte; el servicio al cliente; la selección del local para fabricar y almacenar; el almacenaje; control de inventario; procesamiento de pedidos; traslado de materiales; compras, distribución, abastecimiento de piezas, embalaje, devolución de mercancías; previsión del volumen de pedidos y además de la obligación en las organizaciones de proporcionar a los clientes productos y servicios de la manera más efectiva según sus necesidades y requisitos (Companys Pascual \& Ribas, 2015).

Para Serna Arango et al., (2017) se entiende por indicador una representación cuantitativa verificable en la que se registra, procesa y presenta la información requerida para medir el progreso o descenso de un determinado objetivo. En ese sentido, Forslund (2007) expresa que el uso de indicadores y la gestión del rendimiento en el apartado logístico debe incluir objetivos $\mathrm{y}$ estrategias, en las que se definan indicadores y la cuantificación de estos además del análisis, evaluación y acciones correctivas.

Es de esta manera como estos indicadores de desempeño logístico en la empresa continúan evolucionando para mantener, controlar y desarrollar sus procesos resultando útiles al momento de evaluar y mejorar la relación con los procesos, proveedores y clientes de la organización. Dichos indicadores deben ser; consistentes, dado que debe generarse utilizando la misma fórmula y la misma información, puede ser comparable con el tiempo; Agregables, puesto que deben formular acciones y decisiones que mejoren la calidad de los servicios prestados; y finalmente comparables, es decir que su diseño posibilite el acceso a los mismos datos para que pueda compararse con empresas similares o periodos anteriores.

\section{Metodología}

Tomando en consideración los retos y desafíos que representa el entorno de las organizaciones en la actualidad, se pretenden exponer algunas medidas de desempeño logístico como una panacea desde el ámbito logístico de las organizaciones ante este contexto, además, con el ánimo de darle mayor visibilidad tanto en la academia como en el entorno empresarial a esta temática. Para tales fines se propone una revisión sistemática de la literatura en la cual se busca exponer los aspectos más relevantes asumiendo como objetivo resumir la información existente sobre temas específicos (Manterola et al., 2013).

La revisión de la literatura analítica propuesta incluye búsquedas en bases de datos electrónicas y revistas en línea, dicha investigación filtro los artículos vinculados con la temática en estudio en base a dos criterios. El primero es la relevancia de los modelos logísticos en las bases de datos electrónicas, es decir, que fueran ampliamente citados dentro de Scopus y WOS de tal manera que diversas investigaciones las abordaran y validaran sus proposiciones. El segundo criterio obedece a la pertinencia dentro del uso empresarial, esto significa que se seleccionaron aquellos criterios que contribuyan con los objetivos organizacionales más allá de simplemente realizar un dictamen de la situación en términos logísticos de la organización.

\section{Hallazgos}

\section{Medición de indicadores de desempeño de niveles estratégico, táctico y operativo de Gu- nasekaran.}

Tomando en cuenta que algunas de las medidas y mediciones tradicionales en el ámbito logístico no sean adecuadas para el nuevo entorno en el que muchas actividades no son fácilmente identificables, la medición de los intangibles y las medidas de rendimiento no financieras plantean el mayor desafío en la llamada economía del conocimiento. No obstante, su medición es muy importante para el éxito de las operaciones de las empresas en este entorno, bajo este marco Gunasekaran \& Kobu (2007) proponen una medida que vincule medidas financieras y no financieras como se aprecia la siguiente tabla. 
Tabla 1. Indicadores de desempeño niveles (Estratégicos, tácticos y operativos)

\begin{tabular}{|c|c|c|c|}
\hline Nivel & Indicadores de desempeño & Financiero & No financiero \\
\hline \multirow[t]{2}{*}{ Estratégico } & Tiempo total el flujo del dinero & & $\mathrm{X}$ \\
\hline & Tasa de retorno de la inversión & $\mathrm{X}$ & \\
\hline \multirow{8}{*}{ Táctico } & Flexibilidad de atención a necesidades particulares de clien- & & $\mathrm{X}$ \\
\hline & Tiempo de ciclo de entrega & & $\mathrm{X}$ \\
\hline & Tiempo total de ciclo & & $\mathrm{X}$ \\
\hline & Nivel de relación estratégica Cliente - Proveedor & & $\mathrm{X}$ \\
\hline & Tiempo de entrega al cliente & & $\mathrm{X}$ \\
\hline & Grado de la cooperación para mejorar la calidad & & $\mathrm{X}$ \\
\hline & \multirow[t]{2}{*}{ Costo total de transporte } & & \\
\hline & & $\mathrm{X}$ & \\
\hline \multirow{6}{*}{ Operativo } & Confiabilidad del pronóstico de la demanda & & $\mathrm{X}$ \\
\hline & Tiempo del ciclo de desarrollo del producto & & $\mathrm{X}$ \\
\hline & Costo de manufactura & $\mathrm{X}$ & \\
\hline & Utilización de capacidad & & $\mathrm{X}$ \\
\hline & Costo por información & $\mathrm{X}$ & \\
\hline & Costo por inventario & $\mathrm{X}$ & \\
\hline
\end{tabular}

Fuente: (Angappa Gunasekaran \& Kobu, 2007) Medición de indicadores de desempeño en
los procesos de la cadena logística.

Se pueden determinar muchos indicadores de medición en cada proceso de la cadena logística y estos indicadores se pueden usar para medir los niveles de rendimiento. Esto ha ayudado enormemente a los gerentes y clientes de la compañía a tomar decisiones sobre la optimización de procesos de acuerdo con la siguiente tabla.

\section{Tabla 2. Fases de la cadena de abastecimiento}

Desempeño de la planeación

Desempeño del aprovisionamiento

Desempeño de la producción

Desempeño de la distribución

Nivel de servicio
Ciclo de tiempo para desarrollo del producto.

Método de recepción del pedido

Tiempo total del ciclo de entrega. Seguimiento de la trayectoria del pedido.

Ciclo de flujo del dinero.

Nivel de intercambio de información cliente-proveedor.

Iniciativas de ahorro de costos del cliente y el proveedor.

Extensión de la cooperación mutua para la mejora. Grado de compromiso del proveedor e influencia en las decisiones.

Grado de asistencia mutua para la solución de problemas.

Diversidad de productos y servicios.

Grado de utilización de la capacidad instalada.

Eficiencia de las técnicas de programación.

Costos de manufactura.

Nivel de inventarios.

Tiempo del ciclo del proceso de entrega. Entrega a tiempo.

Número de entregas perfectas.

Flexibilidad para entregas al cliente.

Costo total de distribución.

Flexibilidad.

Tiempo de respuesta a requerimientos de información.

Nivel de servicio comparado con la competencia.

Percepción del servicio por parte del cliente.

Flexibilidad. 
Medición de indicadores mediante el modelo SCOR.

El modelo de SCOR (Supply Chain Operations Reference) permite describir, analizar y optimizar el rendimiento de la cadena de suministro. Desde 1996, el modelo se ha utilizado como estándar para la gestión e integración de diversas cadenas de suministro entre diferentes eslabones de la cadena, y es efectivo en varios departamentos comerciales, organizado en cinco procesos; Planificación, demanda, suministro y gestión de recursos; Suministro, es la estructura básica de suministro y adquisición de materiales, nivel de inventario y gestión de proveedores; Fabricación, elaboración a pedido, fabricación a inventario o fabricación a pedido y actividades de producción; Distribución, gestión de pedidos, Almacenamiento y transporte; devoluciones: gestione las normas comerciales, intercambio de inventario, bienes, transporte y requisitos reglamentarios.

El uso del modelo SCOR como herramienta puede resolver cualquier problema importante en la cadena de suministro como; Optimizar la efectividad de la gestión de la cadena, obteniendo así un servicio al cliente de máxima calidad; Optimizar los costes, al usar métricas para evaluar y comparar diferentes estrategias; Optimizar la gestión de relaciones con proveedores y colaboradores, facilitadas por el uso de un lenguaje común estandarizado; Optimizar la gestión de riesgos de la cadena de suministro, mediante herramientas de planificación, identificación de riesgos y coordinación entre los diferentes eslabones de la cadena; Optimizar la gestión del capital humano, donde la experiencia y el talento es cada vez más importante a medida que las cadenas de suministro se vuelven más complejas y competitivas.

\section{Medición de indicadores mediante el Mod- elo Referencial Logístico.}

El Modelo de referencia de logística (LMR) de Donald Bowersox es una herramienta que puede recopilar todo el concepto que se puede aplicar a la gestión de la cadena de suministro (SCM). El modelo no solo apunta a mejorar la competitividad de la empresa, sino también a mejorar la competitividad general. Cadena de suministro y su sistema logístico. La herramienta consta de trece (13) elementos evaluables en la empresa, numerados de la siguiente manera:

- Concepto sobre logística.

- Organización logística.

- Tecnología de la manipulación.

- Tecnología de almacenaje.

- Tecnología de transporte interno.

- Tecnología de transporte externo.

- Tecnología de comunicaciones.

- Tecnología de software.

- Talento humano.

- Integración de Supply Chain.

- Barreras logísticas.

- Logística reversa.

- Medida de desempeño logístico.

Teniendo en cuenta los modelos e indicadores expuestos anteriormente, se puede concluir que los indicadores logísticos permiten el monitoreo y la planificación de estrategias operativas, que se reflejan en los objetivos propuestos implementados a través del plan de acción.

Igualmente se puede concluir que los indicadores deben ser:

- Sencillos y claros: Que permitan la identificación rápida de las informaciones claves.

- Fáciles y rápidos de elaborar: Con el fin de asegurar su publicación regular.

- Pertinentes: Que contengan los datos relativos a las actividades estudiadas.

- Adaptados a cada función, oficio, proyecto o a cada necesidad. 
Es por esto que se ha determinado para el presente trabajo realizar la medición de los procesos logísticos y administrativos del CROC utilizando la herramienta de Modelo Referencial Logístico, el cual se aplica en función de procesos más precisos y a su vez amplios para toda la cadena de suministro del centro recreacional de oficiales, incluyendo las teorías que a continuación se describen.

\section{Conclusiones y Discusiones.}

Se presentaron los indicadores de medida de desempeño de la cadena logística basados en una búsqueda sistemática que permitió exponer algunos de estos. Bajo esas consideraciones es posible concluir que existen diversidad de indicadores con características que posibilitan su efectividad en casos específicos, por lo tanto, es tarea de quien aplica estos indicadores el ubicar el que responde a las necesidades concretas de la organización, brindando la mayor fiabilidad y aumentando las ventajas competitivas y de esa manera hacer frente a los retos y desafíos de los mercados actuales.

Los resultados del presente artículo hacen un aporte en cuanto a las dinámicas sobre el desempeño logístico de las organizaciones, debi- do a que expone en términos sencillos las principales características de cada una, brindando una visión holística de la temática que será un insumo valioso para que futuros trabajos indaguen acerca de los resultados organizacionales en términos logísticos en otras ramas del pensamiento.

En el campo de las discusiones, se requiere que muchas instancias que no utilizan las medidas de desempeño logístico hagan uso de ellos, esto es; en los centros de educación superior y su posicionamiento en el mercado (Del Rio Cortina et al., 2017); en los estudios de desarrollo económico de territorios (Del Rio Cortina et al., 2019) y finalmente la generación de valor en economías emergentes (Del Río Cortina et al., 2018), de esta manera se podrían promover nuevos niveles de competitividad en las áreas mencionadas y permitir mayores y mejores oportunidades de crecimiento.

\section{Agradecimientos.}

El presente artículo se construyó a partir de los resultados de la tesis de maestría dirigida Luis Guillermo Garcés Henao y de la autoría de los maestrantes Robinson Carvajal González \& Andrés Del Bosque Vega Quintero.

\section{Referencias}

Arango Serna, D. M., Ruiz Moreno, S., Ortiz Vásquez, L. F., \& Zapata Cortes, A. J. (2017). Indicadores de desempeño para empresas del sector logístico: Un enfoque desde el transporte de carga terrestre Performance indicators for logistics enterprises: A land freight transport scope. Revista Chilena de Ingeniería, 25(4), 707-720. http://dx.doi.org/10.4067/S0718-33052017000400707

Companys Pascual, R., \& Ribas, I. (2015). Some Trends and Applications of Operational Research/ Management Science to Operations Management. International Journal of Production Management and Engineering, 3(1), 286-290. https://doi.org/10.4995/ijpme.2015.3459.

Del Rio Cortina, J., Arias, C. R., Espitia, N. L., \& Arbeláez, D. C. (2019). Prospective and territorial development in island areas: Empirical evidence from Bocachica-Colombia. Problems and Perspectives in Management, 17(4), 176-189. https://doi.org/10.21511/ppm.17(4).2019.15

Del Rio Cortina, J., Cardona-Arbelaez, D., \& Simancas-Trujillo, R. (2017). Propuesta de un modelo teórico de branding para el posicionamiento de la marca universitaria. Espacios, 38(53). https://doi.org/https://hdl.handle.net/20.500.12585/8968

Del Río Cortina, J., Cardona Arbelaez, D., \& Pérez Olascuaga, S. J. (2018). Estrategias diferenciadoras y generadoras de valor en economías emergentes. Caso. Saber, Ciencia y Libertad, 13(1), 133-144. https://doi.org/10.18041/2382-3240/saber.2018v13n1.2083 
Del Río Cortina, J., Velasco Alma Rocio, I., Mendoza Jimenez, W., Pérez Narváez, K., Castilla Pue1lo, C., Orozco Barrera, A., \& Herrera Ballesteros, W. (2009). Una Perspectiva De La Logística Desde La Academia. http://www.eumed.net/libros-gratis/2009c/594/index.htm

Fram, T. O. B., \& Licona, J. M. (2016). La logística y la gestión de la información. Revista Cultural Unilibre, (1), 77-90.

Forslund, H. (2007). The impact of performance management on customers' expected logistics performance. International Journal of Operations and Production Management, 27(8), 901-918. https://doi.org/10.1108/01443570710763822

Gunasekaran, A., Patel, C., \& Tirtiroglu, E. (2001). Performance measures and metrics in a supply chain environment. 10.1108/01443570110358468. In International Journal of Operations \& Production Managemen (Vol. 21, Issue 1). https://doi.org/10.1108/01443570110358468

Gunasekaran, Angappa, \& Kobu, B. (2007). Performance measures and metrics in logistics and supply chain management: A review of recent literature (1995-2004) for research and applications. International Journal of Production Research, 45(12), 2819-2840.

Manterola, C., Astudillo, P., Arias, E., \& Claros, N. (2013). Systematic Reviews of the Literature: What Should be Known About Them. Cirugía Española (English Edition), 91(3), 149-155. https://doi.org/10.1016/j.cireng.2013.07.003

Orjuela Castro, J. A., Caicedo-Otavo, A. L., Ruiz-Moreno, A. F., \& Adarme-Jaimes, W. (2016). Efecto de los mecanismos de integración externa en el desempeño logístico de cadenas Frutícolas. Un enfoque bajo dinámica de sistemas. Revista Colombiana de Ciencias Hortícolas, 10(2), 311-322. https://doi.org/10.17584/rcch.2016v10i2.5073

Pinheiro de Lima, O., Breval Santiago, S., Rodríguez Taboada, C. M., \& Follmann, N. (2017). A new definition of internal logistics and how to evaluate it. Ingeniare, 25(2), 264-276. https://doi.org/10.4067/S0718-33052017000200264 